\title{
Implementación de sistema contable en el Restaurante La Terraza Colombiana
}

Geysis Lisbeth Benavides Rodríguez ${ }^{1}$

Delma Lucia Mairena Montenegro²

Juan Carlos Beavides Fuentes ${ }^{3}$

\section{RESUMEN}

Para el desarrollo de la presente investigación se tomó primeramente, aspectos teóricos relacionados con la contabilidad del servicio de restaurante, costo del servicio así como los aspectos que debe incluir el sistema contable a implementar y las formas de validarlo. Después de recopilar la base teórica, se prosiguió a compararlo con la realidad, por lo que se decidió llevar a cabo este estudio en el restaurante la Terraza Colombiana de la ciudad de Estelí. Donde utilizando instrumentos como la entrevista, la observación, se logró identificar la situación contable que presentaba el restaurante. Encontrando debilidades en cuanto al establecimiento del precio del servicio, desconocimiento del costo del servicio, margen de ganancias así como también se pudo comprobar la ausencia de controles para el registro contable.

Palabras Claves: Determinación de los costos del platillo, Sistema contable, Restaurante

Recibido: 15 de diciembre de 2015

Aceptado: 29 de enero de 2016

1 UNAN-Managua/FAREM-Estelí. Correo Electrónico: geysis92@yahoo.com 2 UNAN-Managua/FAREM-Estelí. Correo Electrónico: delma-mairena@outlook.es 3 UNAN-Managua/FAREM-Estelí. Correo Electrónico: juan_carlos0406@yahoo.com 


\section{Implementation of accounting system at the Restaurant "La Terraza Colombiana"}

\section{SUMARY}

For the development of this research, first was taken, the theoretical aspects related to the accounting system of the restaurant, cost of service and the aspects that should include the accounting system to be implemented and the ways to validate it. After collecting the theoretical basis, it was compare with the reality, so it was decided to conduct this study at the Restaurant Terraza Colombiana in the city of Estelí. The data collection techniques were the interview guides and the observation guide, these allowed to identify the accounting situation prevalent in the restaurant. Finding weaknesses in setting the price of the service, lack of knowledge about the service cost, profit margin and the absence of accounting records.

Keywords: Course Costing, Accounting System, Restaurant 


\section{INTRODUCCIÓN}

En Nicaragua, de cada diez personas que trabajan para una empresa, al menos siete aseguran que están en una que no tiene sistema contable, es decir no llevan registros detallados de sus operaciones. Encontrando que muchas de estas empresas son restaurantes.

Tal es el caso que presenta el Restaurante La Terraza Colombiana, mostrando ausencia de un sistema contable que le lleve un riguroso control de todas las operaciones contables que se efectúan y que le brinde información oportuna sobre el desarrollo de su negocio.

Esta situación se presenta en gran medida porque este restaurante lleva sus controles básicos estrictamente sobre la base de lo que dispone el Régimen de cuota fija, en el cual le exige únicamente un cuaderno de ingresos y egresos.

Al no presentar sistemas contables, esto conlleva a graves perjuicios para su negocio. Por lo que el principal objetivo de esta investigación es el de Implementar un sistema contable en el restaurante la Terraza Colombiana, que aporte a la minimización de la inadecuada aplicación de políticas en el establecimiento de precios, falta de información oportuna que contribuya a la toma de decisiones al desconocer los costos reales del servicio que prestan y de su utilidad.

Siendo imprescindible referir la consulta documental de trabajos realizados que guarden relación con los objetivos propuestos en este estudio, como en el caso de Dilman Ponce \& Néstor Mamani (2013) realizó un estudio en lo referente al Control contable del restaurante "Grey Goose". Teniendo como propósito dar a conocer el mejor proceso para el control de los costos del restaurante Grey Goose Arequipa. Del mismo modo Jhader Robles (2007) efectuó un trabajo investigativo sobre Técnicas de la Contabilidad de Costos en las pequeñas y medianas empresas industriales de bienes y servicios del municipio de Juigalpa en el primer semestre del 2007, siendo su principal objetivo Determinar las Técnicas de Contabilidad de Costos en los Hoteles y Restaurantes del Municipio de Juigalpa, en el I semestre del 2007.

\section{MATERIALES Y MÉTODOS}

La orientación del presente estudio, es cualitativo, porque a través de este se pretende fundamentalmente profundizar en las implicaciones que trae la implementación de un sistema contable en un establecimiento que lleva únicamente un sistema de facturación y analizar el comportamiento del fenómeno estudiado, el cual debe ser observado y analizando a profundidad en todo el proceso de acumulación de la información contable, para verificar su funcionalidad en las operaciones contables llevadas por la empresa y analizando los beneficios obtenidos con este.

Es un estudio de caso porque se pretende analizar cómo implementar un sistema contable en la empresa en cuestión, adecuado a los requerimientos del establecimiento.

A partir de esto se pretende ampliar el conocimiento en un entorno real, porque de este modo se puede analizar existencia de problemas en las operaciones de la empresa, determinar el método de análisis a utilizar así como las diferentes alternativas o cursos de acción para resolver la situación encontrada.

Es decir, estudiarlo desde todos los ángulos posibles y por último, establecer conclusiones que se podrían aplicar a diversas empresas dedicadas a prestar un servicio de restaurante.

\section{RESULTADOS}

Al realizar el diagnóstico sobre la situación contable que presentaba el restaurante la Terraza Colombiana, se abordaron los aspectos operativos de las áreas 
principales para la prestación del servicio, como lo es el área de bar y cocina.

Al consultarle a la propietaria sobre el control contable que se lleva en su establecimiento y mediante la observación, se encontraron debilidades y ausencia en los controles, tanto internos como contables.

Respecto al inventario de carnes y bebidas, plantea que estos no son llevados de forma diaria, referente al pago de los colaboradores, ninguno se encuentra actualmente inscrito en el INSS, por lo que no poseen seguro, no se les paga horas extras y tampoco se posee una planilla de pago.

No se contempla la depreciación de los activos fijos, no se llevaba un control de gastos tampoco la llevanza de libros contables y algo muy importante que resaltar, es el desconocimiento del costo del servicio brindado y de las utilidades percibidas.

En relación al establecimiento del costo del servicio que presta el restaurante, este lo estiman haciendo un detalle de la materia prima con la que se elaboran los platillos principales (únicamente los que salen de la carne de res y cerdo) ya que estos son los platillos que más se venden en el restaurante, siendo los que principalmente a su parecer le dejan buenas ganancias. A estos, ella le suma un monto, el cual no lo tienen definido y con el resto de platillos hace lo mismo, es decir solo un estimado, respecto a la mano de obra y otros gastos en los que se incurre para poder llevar a cabo la prestación del servicio del restaurante no son tomados en cuenta como costos del servicio. Resultado de esto se obtiene un costo irreal del servicio, muy por debajo de los que realmente se incurre.

Según lo planteado por la teoría el sistema de costos en los servicios de restaurante, se dan mediante la acumulación de gran cantidad de costos asociados al servicio, por departamento o área de operación, y luego con fines de fijar tarifas y calcular los cobros a los clientes por servicios se realiza una identificación por órdenes del cliente, según la cantidad consumida (platos, bebidas y otros). Específicamente, el costo de los alimentos (material directo) se identifica por plato o servicio consumido por cada cliente mientras que los costos asociados a mano de obra y otros costos generales son identificados e imputados al centro de costos correspondiente, los cuales según Rodríguez (2004), se costean en forma departamental y por totales. Según lo expuesto en las siguientes tablas

\begin{tabular}{llll}
\hline Establecimiento & La Terraza Colombiana & Costos Indirectos & C 42.90 \\
Nombre del Plato & Picada Colombiana especial & T/ Insumos Consumidos & $\mathrm{C} \$ 122.90$ \\
Descripción del Plato & Pollo, Res, Cerdo & Margen de Utilidad & $30 \%$ \\
Porciones & 3 & Precio de Venta & $\mathrm{C} \$ 159.77$ \\
Costo Unitario & $\mathrm{C} \$ 40.97$ & Precio de Venta Unitario & $\mathrm{C} \$ 53.26$ \\
\hline
\end{tabular}

\begin{tabular}{|c|c|c|c|c|c|c|c|c|c|}
\hline Insumos Consumidos & $\begin{array}{c}\text { Unidad de } \\
\text { Medida }\end{array}$ & Cantidad & $\begin{array}{c}\text { Costo } \\
\text { Unitario }\end{array}$ & Total & M.D. & M.O.D. & C.I.F. & Variable & Fijos \\
\hline Pollo & Onzas & 8.00 & $C \$ 3.75$ & $C \$ 30.00$ & C\$ 30.00 & & & $\mathrm{C} \$ 30.00$ & \\
\hline Res & Onzas & 4.00 & C\$ 5.00 & C\$ 20.00 & C\$ 20.00 & & & C\$ 20.00 & \\
\hline Cerdo & Onzas & 4.00 & $C \$ 3.50$ & $C \$ 14.00$ & C\$ 14.00 & & & C\$ 14.00 & \\
\hline Plátano Verde & Unidad & 1.50 & $C \$ 4.00$ & $\mathrm{C} \$ 6.00$ & $\mathrm{C} \$ 6.00$ & & & C\$ 6.00 & \\
\hline Chicharrón & Onzas & 3.00 & C\$ 10.00 & C\$ 30.00 & & & C\$ 30.00 & $\mathrm{C} \$ 30.00$ & \\
\hline Lechuga Nacional & Hojas & 2.00 & $\mathrm{C} \$ 1.50$ & $\mathrm{C} \$ 3.00$ & & & $\mathrm{C} \$ 3.00$ & $\mathrm{C} \$ 3.00$ & \\
\hline Aguacate & Unidad & 0.25 & C\$ 40.00 & C\$ 10.00 & C\$ 10.00 & & & C\$ 10.00 & \\
\hline Gas Butano * & Promedio & 1.00 & C\$ 3.30 & C\$ 3.30 & & & C\$ 3.30 & C\$ 3.30 & \\
\hline
\end{tabular}




\begin{tabular}{|c|c|c|c|c|c|c|c|c|}
\hline Carbón & Promedio & 1.00 & C\$ 3.30 & & & & & \\
\hline Energía Eléctrica * & Promedio & 1.00 & C\$ 3.30 & C\$3.30 & & C\$ 3.30 & C\$ 3.30 & \\
\hline Agua potable * & Promedio & 1.00 & C\$3.30 & $\mathrm{C} \$ 3.30$ & & $C \$ 3.30$ & C\$ 3.30 & \\
\hline $\begin{array}{l}\text { Suministros/ } 10 \% \\
\text { Insumos CIF }\end{array}$ & C\$ 33.00 & & & & & & & \\
\hline Gas Butano * & Promedio CIF & 1.00 & C\$ 3.30 & & & & & \\
\hline Carbón & Promedio CIF & 1.00 & $C \$ 3.30$ & & & & & \\
\hline Energía Eléctrica * & Promedio CIF & 1.00 & $\mathrm{C} \$ 3.30$ & & & & & \\
\hline Agua potable * & Promedio CIF & 1.00 & $\mathrm{C} \$ 3.30$ & & & & & \\
\hline $\begin{array}{l}\text { Total de Insumos } \\
\text { Consumidos }\end{array}$ & & & & C\$ 122.90 & C\$ 80.00 & C\$ 42.90 & C\$ 122.90 & C\$ - \\
\hline $\begin{array}{l}\text { Estructura porcentual } \\
\text { de costo }\end{array}$ & & & & $100.00 \%$ & $65.09 \%$ & $34.91 \%$ & $100.00 \%$ & $0.00 \%$ \\
\hline
\end{tabular}

Distribución del costo de la mano de obra

\begin{tabular}{ccccccc}
\hline Mes & & $\begin{array}{c}\text { Número de Platos } \\
\text { Producidos }\end{array}$ & & $\begin{array}{c}\text { Salario de Personal de } \\
\text { Cocina }\end{array}$ & \multicolumn{2}{c}{ Costo por Unidad } \\
\hline Mes Abril & 451 & & $\mathrm{C} \$$ & $15,000.00$ & $\mathrm{C} \$$ & 33.26 \\
Mes Mayo & 461 & & $\mathrm{C} \$$ & $15,000.00$ & $\mathrm{C} \$$ & 32.54 \\
Mes Junio & 474 & & $\mathrm{C} \$$ & $15,000.00$ & $\mathrm{C} \$$ & 31.65 \\
\hline
\end{tabular}

Por lo que uno de los principales objetivos planteados fue el diseño de un sistema contable adecuado a las necesidades del restaurante la terraza Colombiana.

Después de haber realizado el diagnóstico sobre la situación contable que presentaba el restaurante, se procedió al diseño del sistema, tratando de cubrir las necesidades de control interno como así también en cuanto a los registros de las operaciones llevadas a cabo por el establecimiento.

Incorporando manuales de funciones y procedimientos operativos, catálogo de cuentas para homogenizar el registro contable, el cual se realizó en base al giro de la empresa, tomando en cuenta el tipo de operaciones que se puedan dar en las principales áreas, como lo son el área de bar y cocina. Por lo que se integraron cuentas que son propias de este tipo de empresas, siendo una de estas la cuenta de Inventario, en la que se realizó un desglose de los suministros tanto para el área de bar como en la cocina.
Manuales de cuenta, en este se realizó una descripción de forma detallada de lo que se debe registrar en cada una de las cuentas, los documentos que darán soporte a la transacción, así como lo que representa su saldo. Como en el caso de la cristalería y cubiertos, este activo por lo general sufre una baja por los daños que ocasionan en estos al momento de ser servidos por los meseros, por los comensales cuando los quiebran, cuando son retirados y aún más cuando son lavados. Y se cargan al momento de su reposición.

El manual de funciones para los procedimientos operativos, se diseñó para optimizar el tiempo, recursos físicos y humanos, a través de procedimientos aplicados a las actividades que se desempeñan diariamente en cada área del restaurante. En la que se sugiere usar técnicas básicas de cocina y bar, para que el servicio sea rápido y ordenado, y que no haya ningún tipo de desperdicio en estas dos áreas y así evitar gastos innecesarios. 
Normas de control interno para para proteger los activos. También se incorporaron formularios para llevar de forma ordenada las operaciones dadas de forma diaria y los estados financieros para resumir la información contable del período.

Se verificó que el sistema contable diseñado e implementado en el restaurante fue de gran utilidad e importancia, donde se partió por el registro detallado de los costos y gastos que se incurrían de forma diaria estos se clasificaron y registraron en los comprobantes de diario, los inventarios de carne y de bebidas se controlaron en kardex de manera separada, se dio la acumulación de los costos para determinar el precio del servicio y así conocer la utilidad del ejercicio contemplando la depreciación de los activos fijos mediante las tablas de depreciación, la mano de obra, los materiales, y otros costos indirectos. Dando como resultados a los estados financieros donde toda esta información se vio resumida. La cual se detalla a continuación.

\section{Restaurante La Terraza Colombiana Comprobante de Diario}

\begin{tabular}{|c|c|c|c|c|}
\hline $\begin{array}{c}\text { Comp. No. } \\
01 \\
\end{array}$ & $\begin{array}{r}\text { Descripción de } \\
\text { Pago de Nómina y gastos cor }\end{array}$ & $\begin{array}{l}\text { ante: } \\
\text { ate al mes de Junio }\end{array}$ & & $\begin{array}{c}\text { Fecha } \\
31 / 06 / 2014 \\
\end{array}$ \\
\hline Código & Nombre de la cuenta & Parcial & Debe & Haber \\
\hline 5102 & GASTOS DE VENTA & & $57,749.17$ & \\
\hline 5102.01 & Agua potable & 350.00 & & \\
\hline 5102.02 & Aguinaldo & $2,500.00$ & & \\
\hline 5102.06 & Combustible y lubricantes & 800.00 & & \\
\hline 5102.08 & Depreciaciones & $15,849.17$ & & \\
\hline 5102.09 & Energía Eléctrica & $3,500.00$ & & \\
\hline 5102.19 & Mantenimiento de edificio e instalaciones & $1,000.00$ & & \\
\hline 5102.2 & Mantenimiento de equipo rodante & 500.00 & & \\
\hline 5102.22 & Papelería y útiles de oficina & 100.00 & & \\
\hline 5102.23 & Sueldos & $30,000.00$ & & \\
\hline 5102.24 & Telefonía & 650.00 & & \\
\hline 5102.26 & Vacaciones & $2,500.00$ & & \\
\hline 5106 & GASTOS DE ADMINISTRACIÓN & & $95,233.34$ & \\
\hline 5106.01 & Agua potable & 350.00 & & \\
\hline 5106.02 & Aguinaldo & $2,166.67$ & & \\
\hline 5106.06 & Combustible y lubricantes & 800.00 & & \\
\hline 5106.08 & Depreciaciones & $13,000.00$ & & \\
\hline 5106.09 & Energía Eléctrica & $3,500.00$ & & \\
\hline 5106.19 & Mantenimiento de edificio e instalaciones & $1,000.00$ & & \\
\hline 5106.20 & Mantenimiento de equipo rodante & 500.00 & & \\
\hline 5106.22 & Papelería y útiles de oficina & 100.00 & & \\
\hline 5106.23 & Sueldos & $71,000.00$ & & \\
\hline 5106.24 & Telefonía & 650.00 & & \\
\hline 5106.26 & Vacaciones & $2,166.67$ & & \\
\hline 5107 & GASTOS GENERALES OPERATIVOS & & $8,450.00$ & \\
\hline
\end{tabular}




\begin{tabular}{|c|c|c|c|c|}
\hline 5107.01 & DGI & 700.00 & & \\
\hline 5107.02 & INTUR & $1,200.00$ & & \\
\hline 5107.03 & MINSA & $1,500.00$ & & \\
\hline 5107.04 & IBI & $1,500.00$ & & \\
\hline 5107.05 & Alcaldía & $1,250.00$ & & \\
\hline 5107.06 & Bomberos & $1,500.00$ & & \\
\hline 5107.07 & Policía & 800.00 & & \\
\hline 1101 & EFECTIVO EN CAJA & & & $117,908.33$ \\
\hline 1101.01 & Caja General & $117,908.33$ & & \\
\hline 1212 & DEPRECIACIÓN ACUMULADA & & & $34,190.83$ \\
\hline 1212.01 & De edificios e instalaciones. & $21,666.67$ & & \\
\hline 1212.02 & De mobiliario & $2,682.50$ & & \\
\hline 1212.03 & De equipo & $4,241.67$ & & \\
\hline 1212.04 & De equipo de entretenimiento & $1,266.67$ & & \\
\hline 1212.05 & De equipo rodante & $4,333.33$ & & \\
\hline 2103.00 & GASTOS ACUMULADOS POR PAGAR & & & 9,333.33 \\
\hline 2103.04 & Aguinaldo & $4,666.67$ & & \\
\hline \multirow[t]{2}{*}{2103.06} & Vacaciones & $4,666.67$ & & \\
\hline & & Totales & $161,432.50$ & $161,432.50$ \\
\hline
\end{tabular}

\section{Restaurante La Terraza Colombiana \\ Estado de Resultados \\ Del 01 de Abril al 30 de Junio de 2014 \\ Expresado en córdobas C\$}

4101 INGRESOS POR EL SERVICIO RESTAURANTE

C\$ 1492,095.20

51 COSTOS Y GASTOS

5101 COSTO DEL SERVICIO

C\$ 220,559.08

5102 GASTOS DE VENTA

Total gasto de venta

$249,465.13$

5106 GASTOS DE ADMINISTRACIÓN

Total gasto de administración

$294,481.13$

5107 GASTOS GENERALES OPERATIVOS

5110 OTROS GASTOS

Total gastos operativos

$25,350.00$

$5,428.50$

TOTAL COSTOS Y GASTOS DEL SERVICIO 


\section{Restaurante La Terraza Colombiana Balance General \\ Del 01 de Abril al 30 de Junio de 2014 Expresado en córdobas C\$}

\begin{tabular}{|c|c|c|c|c|c|}
\hline 1 & ACTIVO & & 2 & PASIVO & \\
\hline 11 & ACTIVO CIRCULANTE & & 21 & PASIVO CIRCULANTE & \\
\hline 1101 & EFECTIVO EN CAJA & $900,116.49$ & 2103 & $\begin{array}{l}\text { GASTOS ACUMULADOS } \\
\text { POR PAGAR }\end{array}$ & $28,000.00$ \\
\hline 1104 & TARJETAS DE CRÉDITO & $565,176.91$ & \multicolumn{2}{|c|}{ Total Pasivos } & $28,000.00$ \\
\hline 1106 & INVENTARIO & $312,707.96$ & 3 & CAPITAL & \\
\hline \multicolumn{2}{|c|}{ Total activo circulante } & $1778,001.36$ & 31 & PATRIMONIO & \\
\hline & & & 3101 & APORTES DE CAPITAL & $5114,615.00$ \\
\hline 12 & ACTIVO FIJO & & & & \\
\hline 1201 & TERRENO & $1300,000.00$ & 3105 & $\begin{array}{l}\text { RESULTADOS DEL } \\
\text { EJERCICIO }\end{array}$ & \\
\hline 1202 & $\begin{array}{l}\text { EDIFICIOS E } \\
\text { INSTALACIONES }\end{array}$ & $2600,000.00$ & 3105 & Utilidad o Pérdida del Período & $696,811.36$ \\
\hline 1203 & MOBILIARIO & $154,360.00$ & & Total Capital & $5811,426.36$ \\
\hline 1204 & EQUIPO & $101,500.00$ & & & \\
\hline 1204.03 & $\begin{array}{l}\text { EQUIPO DE } \\
\text { ENTRETENIMIENTO }\end{array}$ & $79,000.00$ & & & \\
\hline 1210 & EQUIPO RODANTE & $442,000.00$ & & & \\
\hline 1212 & $\begin{array}{l}\text { DEPRECIACIÓN } \\
\text { ACUMULADA }\end{array}$ & $(615,435.00)$ & & & \\
\hline \multicolumn{2}{|c|}{ Total activo fijo } & $4061,425.00$ & & & \\
\hline \multicolumn{2}{|c|}{ TOTAL ACTIVO } & $5839,426.36$ & \multicolumn{2}{|c|}{ TOTAL PASIVO+CAPITAL } & $5839,426.36$ \\
\hline Elaborad & Revisado por & Autc & do por & & \\
\hline
\end{tabular}




\section{CONCLUSIONES Y RECOMENDACIONES}

En base a los objetivos planteados y al análisis realizado, se encontraron las siguientes conclusiones:

El restaurante posee debilidades y ausencia en los controles internos y contables, con respecto al inventario de carnes, bebidas, pago a empleados, protección de los activos, segregación de funciones, deterioro de los activos fijos, control de gastos, llevanza de libros contables.

Ocasionándole desconocimiento del costo del servicio brindado y de las utilidades percibidas.

El sistema diseñado cumple con los requerimientos necesarios y es factible implementarlo.

Por lo que se le recomienda

- Llevar por separado los gastos personales de la propietaria de los gastos que se incurren en el restaurante.

- Crear la cuenta de banco en el catálogo de cuentas.

- Realizar depósitos bancarios en concepto de las ventas, de forma diaria.

- Contratar a una persona que se dedique exclusivamente a la parte contable.

- Inscribir a los trabajadores al seguro social para que gocen de los beneficios que este brinda.

\section{BIBLIOFRAFÍA DE REFERENCIA}

Albert, A. (2010). Scrib.com. Obtenido de Csoto del servicio de alimentos y bebidas: http://es.scribd. com/doc/40490330/Costos-del-servicio-dealimentos-y-bebidas-en-establecimientos-de- alojamientos-turisticos

Cruz, S. (2012). Monografias.com. Obtenido de Sistema de contabilidad: http://www.monografias. com/trabajos93/sistema-de-contabilidad/sistemade-contabilidad.shtml

Escobar, F., \& Toledo, A. (2010). servicios de restaurante. Obtenido de Como opera un restaurante: http://www.trabajo.com.mx/como_ debe_operar_un_restaurante.htm

Horngren, H. (2003). Contabilidad. Mexico: E-books.

Inide. (s.f.). Obtenido de http://www.inide.gob.ni/ ECH/Informe\%20ECH.pdf

Jirón, O. (2014). INTUR, representante. (D. Mairena, Entrevistador)

Lorente, H. (2010). Turismo. Obtenido de Restaurante: http://www.consumoteca.com/turismo-y-viajes/ restauracion/restaurante/

Narváez, S. A., \& Narváez, R. J. (2010). Contabilidad I 4ta Edición. México: Goobooke imprenta.

Negocios. (2014). Como llevar la contabilidad de un restaurante. Obtenido de http://negocios.uncomo. com/articulo/como-llevar-la-contabilidad-de-unrestaurante-21906.html

Noguera, A., \& Vilchez, H. (2010). Nociones basicas de restauranteria. Obtenido de Clasificacion de los restaurantes: http://tallerdeturismopractico2. blogspot.com/p/clasificacion-de-los-restaurantes. html

Orellana, G. L. (2010). Manual de procedimientos operativos. Obtenido de http://repositorio.uct.edu. ec/bitstream/123456789/347/1/Manual\%20de\%20 Procedimiento

Periu, M. (10 de 2012). Finanazas . Obtenido de https:// es-us.finanzas.yahoo.com/blogs/tu-seguro/cuantose-debe-dar-propina-162623466.html?page=all 